\title{
Methylome and transcriptome profiles in three yak tissues revealed that DNA methylation and the transcription factor ZGPAT co-regulate milk production
}

Jinwei Xin ${ }^{1,2 \dagger}$, Zhixin Chai ${ }^{3 \dagger}$, Chengfu Zhang ${ }^{1,2 \dagger}$, Qiang Zhang ${ }^{1,2}$, Yong Zhu ${ }^{1,2}$, Hanwen Cao ${ }^{1,2}$, Cidan Yangji ${ }^{1,2}$, Xiaoying Chen ${ }^{1,2}$, Hui Jiang ${ }^{1,2}$, Jincheng Zhong ${ }^{3^{*}}$ and Qiumei $\mathrm{Ji}^{1,2^{*}}$

\begin{abstract}
Background: Domestic yaks play an indispensable role in sustaining the livelihood of Tibetans and other ethnic groups on the Qinghai-Tibetan Plateau (QTP), by providing milk and meat. They have evolved numerous physiological adaptations to high-altitude environment, including strong blood oxygen transportation capabilities and high metabolism. The roles of DNA methylation and gene expression in milk production and high-altitudes adaptation need further exploration.

Results: We performed genome-wide DNA methylome and transcriptome analyses of breast, lung, and biceps brachii muscle tissues from yaks of different ages. We identified 432,350 differentially methylated regions (DMRs) across the age groups within each tissue. The post-mature breast tissue had considerably more differentially methylated regions $(155,957)$ than that from the three younger age groups. Hypomethylated genes with high expression levels might regulate milk production by influencing protein processing in the endoplasmic reticulum. According to weighted gene correlation network analysis, the "hub" gene ZGPAT was highly expressed in the postmature breast tissue, indicating that it potentially regulates the transcription of 280 genes that influence protein synthesis, processing, and secretion. The tissue network analysis indicated that high expression of HIF1A regulates energy metabolism in the lung.
\end{abstract}

Conclusions: This study provides a basis for understanding the epigenetic mechanisms underlying milk production in yaks, and the results offer insight to breeding programs aimed at improving milk production.

Keywords: Milk production, DNA methylation, Transcription factor, Epigenetic regulation

\footnotetext{
*Correspondence: zhongjincheng518@126.com; jiqiumei07@163.com

†Jinwei Xin, Zhixin Chai and Chengfu Zhang contributed equally to this work.

${ }^{3}$ Key Laboratory of Qinghai-Tibetan Plateau Animal Genetic Resource Reservation and Utilization, Sichuan Province and Ministry of Education, Southwest Minzu University, Chengdu, Sichuan, China

${ }^{1}$ State Key Laboratory of Hulless Barley and Yak Germplasm Resources and Genetic Improvement, Lhasa, Tibet, China

Full list of author information is available at the end of the article
}

(C) The Author(s). 2020 Open Access This article is licensed under a Creative Commons Attribution 4.0 International License, which permits use, sharing, adaptation, distribution and reproduction in any medium or format, as long as you give appropriate credit to the original author(s) and the source, provide a link to the Creative Commons licence, and indicate if changes were made. The images or other third party material in this article are included in the article's Creative Commons licence, unless indicated otherwise in a credit line to the material. If material is not included in the article's Creative Commons licence and your intended use is not permitted by statutory regulation or exceeds the permitted use, you will need to obtain permission directly from the copyright holder. To view a copy of this licence, visit http://creativecommons.org/licenses/by/4.0/ The Creative Commons Public Domain Dedication waiver (http://creativecommons.org/publicdomain/zero/1.0/) applies to the data made available in this article, unless otherwise stated in a credit line to the data. 


\section{Background}

Domestic yaks play an indispensable role in sustaining the livelihood of Tibetans and other ethnic groups on the Qinghai-Tibetan Plateau (QTP), in the Himalayas, and in the connecting Central Asian highlands. They provide milk, meat, hides, fiber, fuel, and transportation $[1,2]$. Yak milk is not only an important source of highquality protein, especially containing high quantities of essential amino acids, but also rich in immunoglobulins, antimicrobial peptides and growth factors [3, 4]. Protein content and composition are essential for the transformation of milk into cheese and other milk-derived products, and therefore important for the dairy industry. Previous researches indicated that manipulation of DNA transcription and posttranscriptional regulation can improve the efficiency of milk production. The synthesis of fat, protein, and lactose in milk can be regulated by the activity of specific transcription factors, noncoding RNAs, and alterations of the chromatin structure in mammary epithelial cells [5]. Despite such advancements in research, little is currently known about the physiological and cellular regulation required for milk protein synthesis and secretion in yak. We hypothesized that the genes responsible for milk production were regulated by DNA methylation and that distinct sub-modules of correlated expression variation could be identified. In this study, we performed genome-wide DNA methylome and transcriptome analyses of yak lung, breast, and biceps brachii muscle tissues at four different stages of development to identify the regulatory networks associated with milk protein synthesis, metabolism, and secretion.

\section{Results}

\section{Global DNA methylation and gene expression in the} breast, lungs, and biceps brachii muscle at different ages We generated the methylomes and transcriptomes of lung, breast, and biceps brachii muscle tissues from 12 female Riwoqe yaks at four different stages $(n=3 /$ stage) of development: 6 months old (MO) (young), $30 \mathrm{MO}$ (pre-mature), 54 MO (mature) and $90 \mathrm{MO}$ (post mature). Among these, only the post-mature yaks (90 months) were lactation, with $\sim 3.16 \mathrm{~kg} /$ day milk yield. After performing sequence quality control and filtering, we obtained a single-base resolution methylome covering $85.6 \%(27,471,373 / 32,092,725)$ of CpG sites across the genome with an average depth of $22.5 \times$. We first calculated pairwise Pearson's correlations of CpG sites with at least $10 \times$ coverage depth across all samples, which were well clustered by tissue types (Fig. 1a). The correlation of CpG methylation levels for biological replicates was strong (median Pearson's $r=0.74$ ), the correlation of CpG methylation levels between ages (median Pearson's $r=0.72$ ) was relatively weaker and the correlation between tissues was weakest (median Pearson's $r=0.66$ ) (Fig. 1c). We aligned the transcriptome sequencing data for all samples to our newly assembled yak genome reference, and we subsequently obtained the transcripts. In total, we obtained 20,504 transcripts, that were then annotated to the Gene Ontology (GO) [6], InterPro [7], Kyoto Encyclopedia of Genes and Genomes (KEGG) [8], Swiss-Prot [9], and TrEMBL [10] databases (Table S1). We also calculated pairwise Pearson's correlations of all transcripts and obtained similar results to those of DNA methylation (Fig. 1b). Biological replicates showed the highest correlation coefficients, while different tissues showed the lowest correlation coefficients (Fig. 1d).

\section{Differentially methylated regions among the age groups}

We determined differentially methylated regions (DMRs) across age groups within the breast, lung, and biceps brachii muscle tissues (Table S2, S3, S4). Within the lung and biceps brachii muscle tissues, age groups did not differ in age-related DMRs (A-DMRs), but post-mature breast tissue had considerably more DMRs $(155,957)$ than the three younger age groups (Fig. 2a). We then investigated the correlations between DMRs and their corresponding differentially expressed genes. The ratio of negatively to positively correlated gene pairs was 1.02 for promoters with DMRs and 0.95 for gene bodies with DMRs. Not every methylation was correlated with the expression of its associated gene, due to the gene regulation complexity [11].

At $\sim 90$ months, $\sim 120$ days after giving birth for the third time, yaks are in the lactation period (milk yield of $\sim 3.16 \mathrm{~kg} /$ day), so it is possible that the observed methylation partially controls yak lactation. Since promoter methylation decreases gene expression [11], we selected 375 hypomethylated promoter genes (highly expressed) along with 207 hypermethylated promoter genes (lowly expressed) from post-mature yak breast tissues. The hypomethylated (highly expressed) genes were only enriched in "protein processing in endoplasmic reticulum (ER)" (9 genes, 2.964-fold enrichment, $p=$ 0.0049). Specifically, the genes are involved in vesicle trafficking (SEC23B), oligosaccharide linking (MOGS, RPN2), folding and assembly (HSPA5), transportation (LMAN2, SEL1L), and ubiquitination and degradation (UBE2J1, UBE2J2, DERL2) [12, 13]. These genes were significantly upregulated at 90 months in breast tissues, but not in lung and biceps brachii muscle tissues (Fig. 2b). Based on this data, methylation might help regulate milk production by influencing protein processing in the endoplasmic reticulum during the lactation period.

We also examined A-DMRs that overlapped across age groups. Young and post-mature tissues rarely shared A-DMRs when comparing the lung and muscle tissues (for young tissues, muscle: 586 A-DMRs, breast: 2249 ADMRs, lung: 496 A-DMRs; for post-mature tissues, 

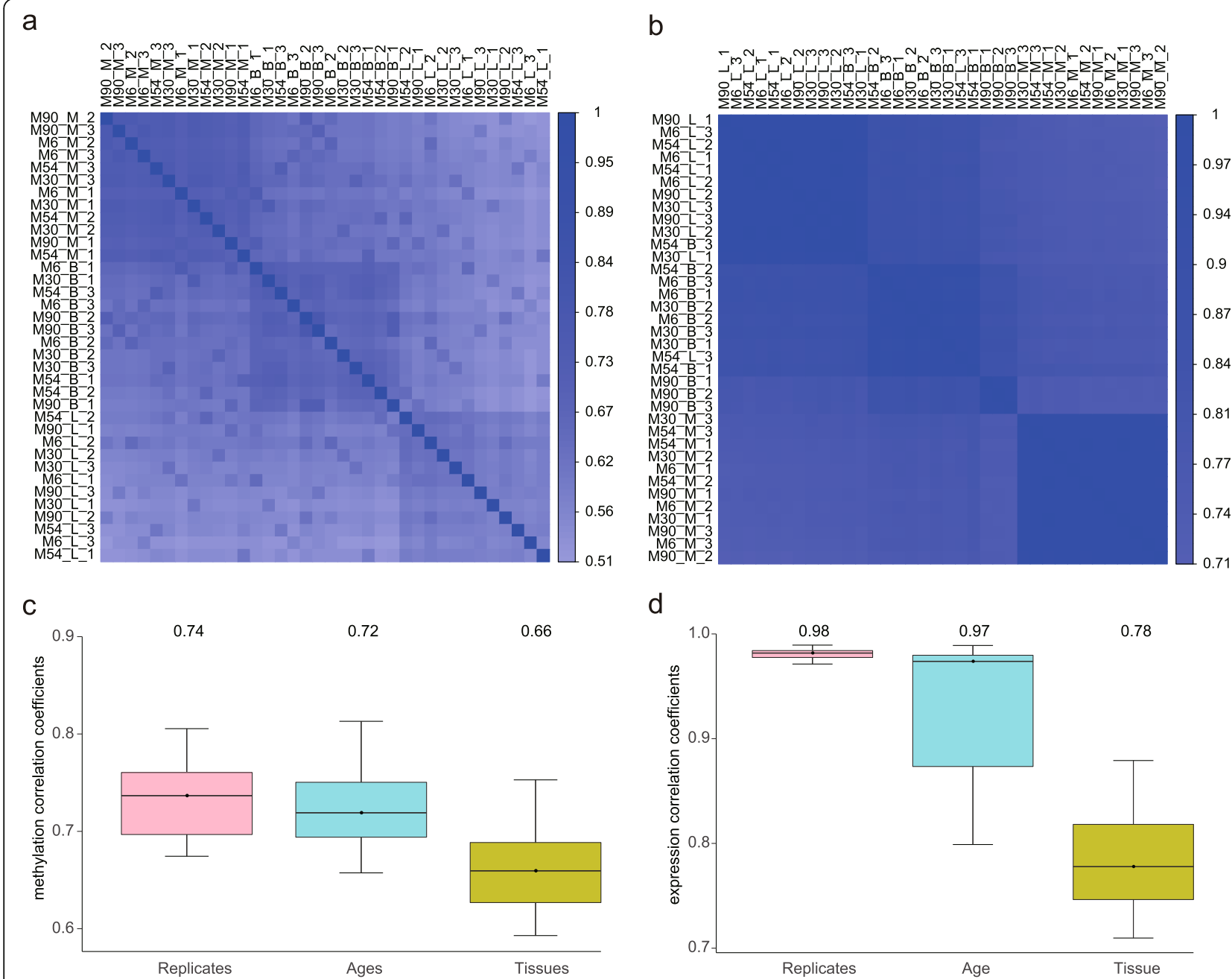

d

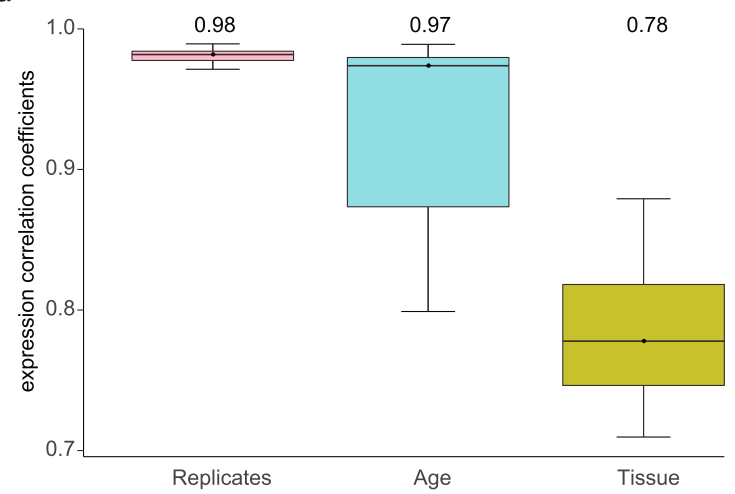

Fig. 1 Global DNA methylation and gene expression among samples. Pearson's correlation analysis based on the methylation of CpG sites (a) and gene expression (b) among samples. Boxplot of Pearson's correlation coefficients between replicates, ages, or tissues for methylation (c) and gene expression (d). M6, M30, M54 and M90 represent different 6, 30, 54 and 90 months old, respectively. B, L, and M represent breast, lung, and biceps brachii muscle, respectively. 1, 2, and 3 represent different replicates

muscle: 470 A-DMRs, breast: 12,050 A-DMRs, lung: 772 A-DMRs) (Fig. 2c, d). Pre-mature and mature stages also rarely shared A-DMRs across muscle and lung tissues (Fig. 2e), suggesting that methylation patterns were already established at the young stage and that no extensively divergent epigenetic difference occurred across different age groups under natural high-altitude conditions.

\section{Consensus network analysis for tissues and age groups}

We first performed a multi-way ANOVA test for each gene across all samples $(n=36)$ to test the null hypothesis that the gene expression level did not differ among age groups and tissues. At the threshold for significance $(p<0.05), 417$ age-related and 8560 tissue-related genes were selected for further weighted gene correlation network analysis (WGCNA), which uses network topography to group genes into modules based on correlations [14]. Next, we conducted WGCNA for tissue- and age-related gene expression respectively to identify a "consensus network"-a common pattern of genes that are correlated in all conditions. We performed a consensus network, module statistic, and eigengene network analyses to identify modules, to assess relationships between modules and traits, and to study the relationships between co-expression modules [15]. The consensus networks identified for tissues and age groups had clearly delineated modules (Fig. 3a, b), and the modules identified were significantly correlated with tissues and age groups (Fig. 3c, d).

\section{Age network analysis indicates that ZGPAT might regulate milk production}

Within the age-related gene network, the largest module ("turquoise", $n=356$ ) had negative correlation for breast tissue $(r=-0.57, p=3 \mathrm{e}-04)$ and age $(r=0.37, p=0.03)$ 


a
\begin{tabular}{|c|c|c|c|c|}
\hline Breast & M06 & M30 & M54 & M90 \\
\hline M06 & 0 & 20,952 & 23,868 & 62,576 \\
\hline M30 & 20,952 & 0 & 12,347 & 46,898 \\
\hline M54 & 23,868 & 12,347 & 0 & 46,483 \\
\hline M90 & 62,576 & 46,898 & 46,483 & 0 \\
\hline
\end{tabular}

\begin{tabular}{|c|c|c|c|c|}
\hline Lung & M06 & M30 & M54 & M90 \\
\hline M06 & 0 & 22,108 & 22,580 & 27,140 \\
\hline M30 & 22,108 & 0 & 20,208 & 22,231 \\
\hline M54 & 22,580 & 20,208 & 0 & 22,060 \\
\hline M90 & 27,140 & 22,231 & 22,060 & 0 \\
\hline
\end{tabular}
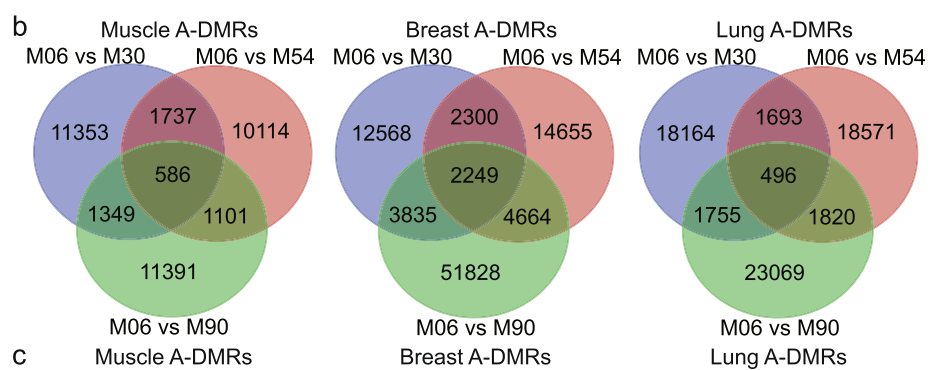

M06 vs M90 M30 vs M90 M06 vs M90 M30 vs M90 M06 vs M90 M30 vs M90

\begin{tabular}{|c|c|c|c|c|}
\hline Muscle & M06 & M30 & M54 & M90 \\
\hline M06 & 0 & 15,025 & 13,538 & 14,427 \\
\hline M30 & 15,025 & 0 & 11,688 & 12,339 \\
\hline M54 & 13,538 & 11,688 & 0 & 15,882 \\
\hline M90 & 14,427 & 12,339 & 15,882 & 0 \\
\hline
\end{tabular}
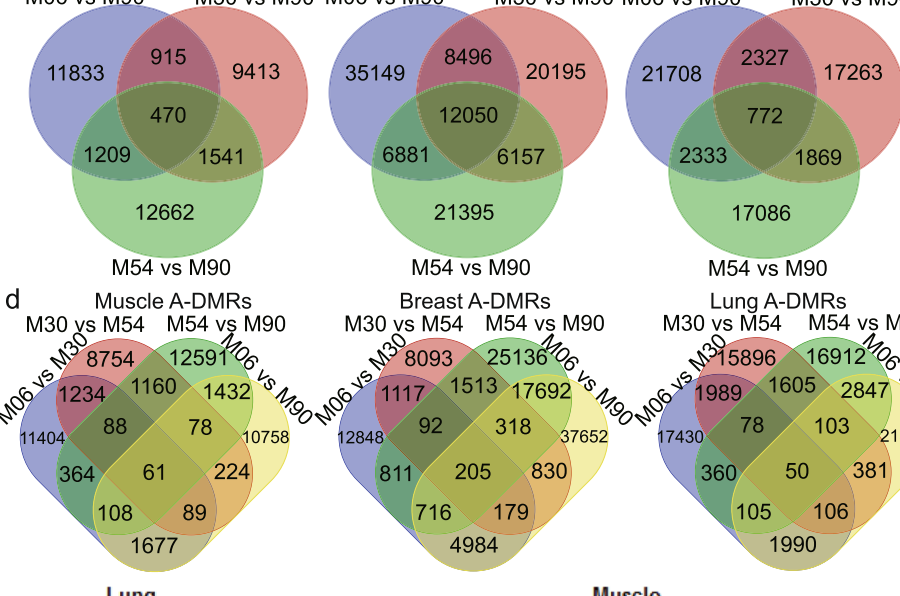

Breast A-DMRs

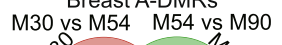

M54 vs M90

Lung A-DMRs

M30 vs M54 M54 vs M90 M30 vs M54 M54 vs M90
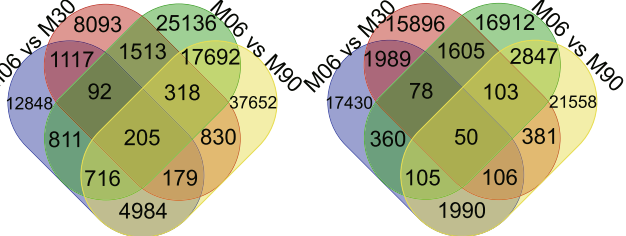

e

Breast

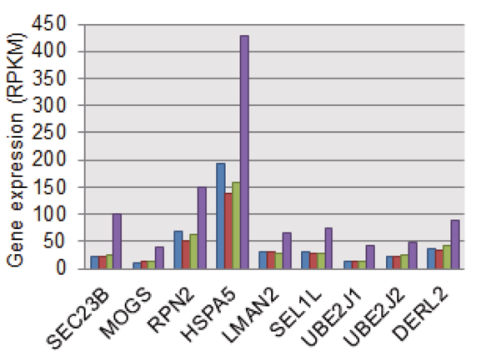

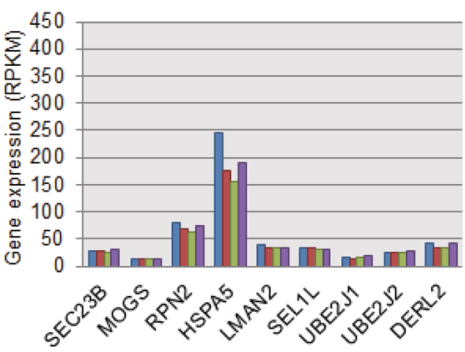

Muscle

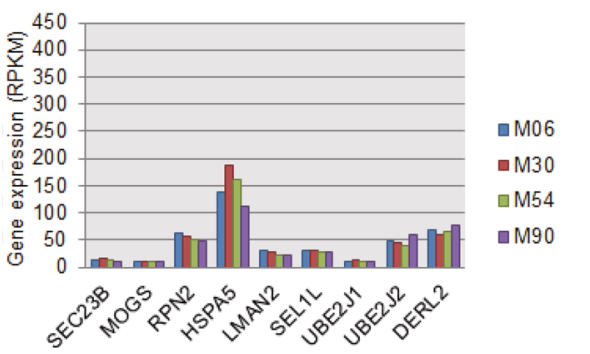

Fig. 2 Overview of age-associated DMRs. a Basic statistics for A-DMRs within each tissue. $\mathbf{b}$ Expression levels of 9 enriched genes in "protein processing in endoplasmic reticulum (ER)". Overlap of A-DMRs associated with the 6-month group (c), 90-month group (d), and 30- and 54months groups (e) in the muscle, breast, and lung respectively

and a positive correlation for biceps brachii muscle tissues (Table S5). The breast tissue had a stronger signal than that of age and may have overwhelmed the signal from age. Genes in this module were enriched in the GO categories of "protein polyubiquitination," "RNA polymerase II core promoter proximal region sequencespecific DNA binding," "ATP binding," "transcription, DNA-templated," and "negative regulation of transcription from RNA polymerase II promoter" ( $p$-values of $0.000344,0.000822,0.000991,0.00139$, and 0.00244 , respectively). The "blue" $(n=48)$ and "grey" $(n=13)$ modules showed only a negative correlation with age $(r=-$ $0.58, p=2 \mathrm{e}-04 ; r=-0.76, p=6 \mathrm{e}-08$, respectively) and exhibited no enrichment of GO categories for genes. After applying the threshold of the absolute value of gene significance for age $(|G S|>0.5)$ and module membership measures $(|\mathrm{MM}|>0.6)$ in each module, we defined 20 and 7 "hub" genes in the "turquoise" and "blue" modules (Table 1). The gene expression of the "hub" genes was well clustered by modules, which was consistent with the negative correlation with age ("turquoise" $r=0.37$, "blue" $r=-0.58$ ). The upregulated expression level of the "hubs" in breast tissue at 90 months old indicated that the "turquoise" module had a stronger correlation with breast tissue than with age (Fig. 4a).

We used the AnimalTFDB 3.0 database [16] to examine transcription factors in these 27 "hubs" and found that ZGPAT encodes a transcription regulator protein and was significantly upregulated in breast tissue at 90 months of age (Fig. 4a). Previous study reported that this protein specifically binds the $5^{\prime}$-GGAG [GA] A [GA]A3' consensus sequence and represses transcription by 


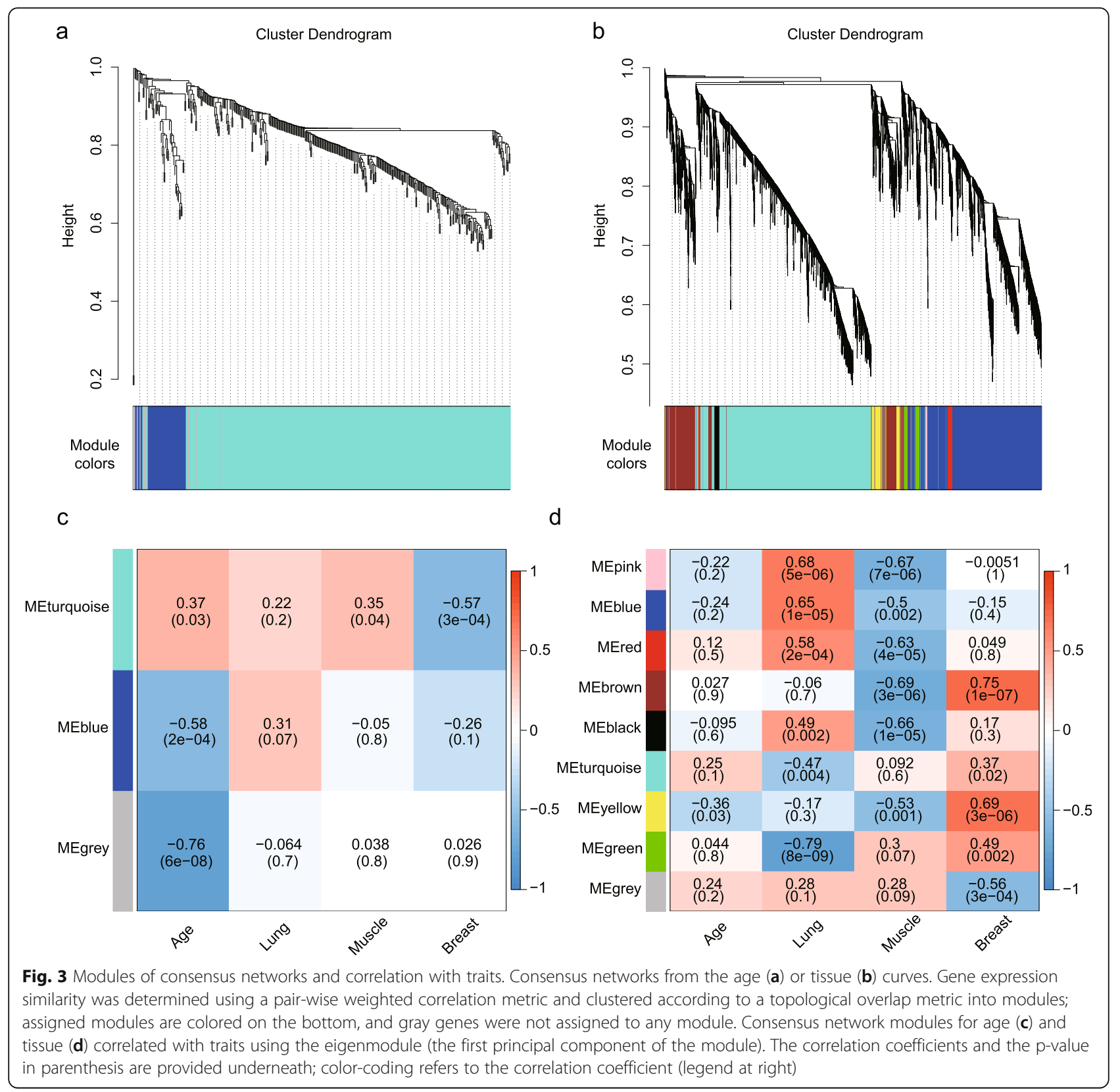

recruiting the chromatin multi-complex NuRD to target promoters [17]. ZGPAT was highly expressed in breast tissue at 90 months, and it potentially regulated the transcription of 280 genes (weight $>0.15$ ) in the network from the "turquoise" module. In order to identify the most important cellular activities controlled by this TF regulatory network, we analyzed over-represented GO biological process and molecular function terms, as well as KEGG pathways. These potential target genes were enriched in the GO categories of "protein binding," "ATP binding," and "zinc ion binding," among others, and the KEGG categories of "aminoacyl-tRNA biosynthesis," "autophagy animal," and "protein processing in endoplasmic reticulum" (Fig. 4b). These enriched GO terms and KEGG pathways likely help regulate protein synthesis, processing, and secretion in breast tissue. For example, 6 of 7 genes from the "protein processing in endoplasmic reticulum" category were also upregulated at 90 months of age in breast tissue (Fig. 4c) and involved in multiple processes in the endoplasmic reticulum, including vesicle trafficking (SEC24C), folding and assembly (SELENOS), transportation (BCAP31), and 
Table 1 List of "hub" genes in the consensus network for age

\begin{tabular}{|c|c|c|c|c|c|c|}
\hline Yak ID & Gene symbol & Module color & Gene significance & $p$-value & Module membership & $p$-value \\
\hline BmuPB009868 & AP3D1 & turquoise & 0.513965075 & 0.001344116 & 0.868945999 & $6.37 \mathrm{E}-12$ \\
\hline BmuPB004083 & TMEM30A & turquoise & 0.505722627 & 0.001652657 & 0.848054843 & $6.65 \mathrm{E}-11$ \\
\hline BmuPB000726 & DDRGK1 & turquoise & 0.53596122 & 0.000754414 & 0.842070133 & $1.22 \mathrm{E}-10$ \\
\hline BmuPB019011 & OTUD3 & turquoise & 0.543916749 & 0.000606215 & 0.828680521 & 4.37E-10 \\
\hline BmuPB000091 & CNPPD1 & turquoise & 0.51195228 & 0.001414359 & 0.814537409 & $1.50 \mathrm{E}-09$ \\
\hline BmuPB006815 & TOR1AIP1 & turquoise & 0.544705745 & 0.000593032 & 0.809789221 & 2.21E-09 \\
\hline BmuPB007137 & MGAT4A & turquoise & 0.545969144 & 0.000572452 & 0.798170686 & $5.51 \mathrm{E}-09$ \\
\hline BmuPB007385 & HECA & turquoise & 0.603273223 & 9.84E-05 & 0.770629907 & 3.85E-08 \\
\hline BmuPB019443 & SYAP1 & turquoise & 0.500377628 & 0.001884511 & 0.733120646 & $3.68 \mathrm{E}-07$ \\
\hline BmuPB009825 & PHAX & turquoise & 0.561938984 & 0.00036184 & 0.70497442 & $1.59 \mathrm{E}-06$ \\
\hline BmuPB008032 & UBE2G2 & turquoise & 0.52385538 & 0.001041698 & 0.70208134 & $1.83 \mathrm{E}-06$ \\
\hline BmuPB012517 & SYF2 & turquoise & 0.568328068 & 0.000299194 & 0.699367268 & $2.08 \mathrm{E}-06$ \\
\hline BmuPB001610 & FURIN & turquoise & 0.567906296 & 0.000303008 & 0.697046314 & $2.32 \mathrm{E}-06$ \\
\hline BmuPB000679 & ZGPAT & turquoise & 0.504624296 & 0.001698141 & 0.683958391 & 4.25E-06 \\
\hline BmuPB007049 & SKP2 & turquoise & -0.527608908 & 0.000943732 & -0.669790419 & 7.91E-06 \\
\hline BmuPB000224 & C2orf6 & turquoise & 0.531191165 & 0.000857925 & 0.652871802 & $1.59 \mathrm{E}-05$ \\
\hline BmuPB007420 & TAB2 & turquoise & 0.505905132 & 0.001645204 & 0.652844304 & $1.59 \mathrm{E}-05$ \\
\hline BmuPB018569 & ORAOV1 & turquoise & 0.51035386 & 0.001472421 & 0.637043276 & 2.95E-05 \\
\hline BmuPB010550 & CCPG1 & turquoise & 0.644779605 & $2.19 \mathrm{E}-05$ & 0.612874899 & 7.08E-05 \\
\hline BmuPB012521 & TMEM57 & turquoise & 0.57317319 & 0.000258349 & 0.60967979 & 7.91E-05 \\
\hline BmuPB013324 & MCM3 & blue & -0.583585649 & 0.000186982 & 0.841505398 & 1.29E-10 \\
\hline BmuPB010064 & SPC24 & blue & -0.509962181 & 0.001486965 & 0.744497748 & $1.93 \mathrm{E}-07$ \\
\hline BmuPB003102 & C17orf49 & blue & -0.526803776 & 0.000964031 & 0.741805787 & $2.26 \mathrm{E}-07$ \\
\hline BmuPB015902 & SERPINH1 & blue & -0.607769763 & $8.44 \mathrm{E}-05$ & 0.721017606 & 7.04E-07 \\
\hline BmuPB016582 & SRPX2 & blue & -0.51838468 & 0.001200558 & 0.698151166 & 2.20E-06 \\
\hline BmuPB012996 & UCK2 & blue & -0.588274454 & 0.000161067 & 0.677420455 & $5.68 \mathrm{E}-06$ \\
\hline BmuPB015372 & UMPS & blue & -0.503577413 & 0.001742514 & 0.648111658 & $1.92 \mathrm{E}-05$ \\
\hline
\end{tabular}

ubiquitination and degradation (BAG1, UBE2G2, and MARCH6) $[12,13]$. Only DNAJC10 was downregulated at 90 months of age in breast tissue. This gene encodes an endoplasmic reticulum co-chaperone that is part of the endoplasmic reticulum-associated degradation complex involved in recognizing and degrading misfolded proteins [13].

\section{Tissue network analysis indicates regulative role of HIF1A} in lung

Within the tissue-related gene module network, four modules showed a positive correlation and two showed a negative correlation with the lung; all significant moduletrait relationships were negative in the muscle but positive in the breast (Fig. 3d). Moreover, 99.54\% of the total 8560 tissue-related genes were related to the top 4 modules ("turquoise," $n=3833$; "blue," $n=2795$; "brown," $n=1052$; "yellow," $n=339$ ) (Fig. 5a, Table S6), and these modules were also highly correlated with other modules; for example, "brown," "yellow," and "black" showed a high eigengene adjacency with each other (Fig. 5b).

We applied the more stringent threshold absolute value of gene significance for the age and module membership measures in the top four modules to identify "hub" genes in the "turquoise," "blue," "brown," and "yellow" modules. With the threshold values of $|\mathrm{GS}|>0.7$ and $|\mathrm{MM}|>0.8$, 34 "hub" genes were identified in the "turquoise" module. Twenty-four hubs were then filtered from the gene significance of module-lung relationships, and 10 were filtered from the gene significance of module-breast relationships (Table 2). These were further divided into 3 clusters by hierarchical clustering, which showed high expression levels in the breast (cluster 1), lung (cluster 2), and biceps brachii muscle (cluster 3 ) tissues, with distinct clustering patterns by tissue (Fig. 5c).

According to the AmalTFDB 3.0 database [16], EBF3, HIF1A, and STAT6 were annotated as transcription factors. EBF3 encodes a member of the early B-cell factor 


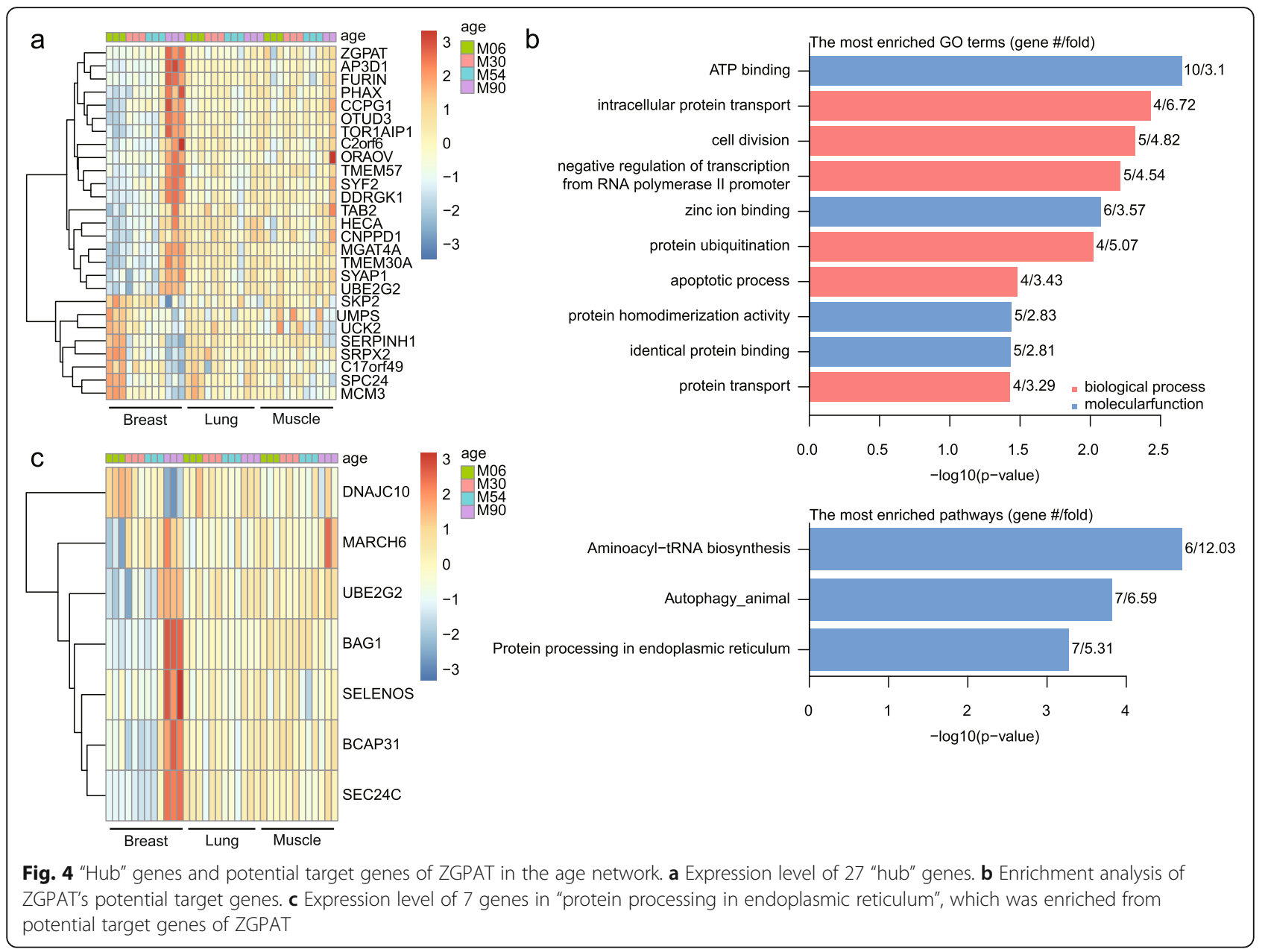

(EBF) family of DNA binding transcription factors, and may function as a tumor suppressor in several types of cancer [18]. STAT6 is a member of the STAT family of transcription factors, which form homo- or heterodimers that translocate to the cell nucleus where they act as transcription activators [19]. HIF1A, hypoxia-inducible factor-1, functions as a master transcriptional regulator of the cellular and systemic homeostatic response to hypoxia [20]. HIF1A was upregulated in the lung to potentially control the transcription of 2008 genes (weight $>0.15$ ), which were enriched in multiple GO biological process categories, molecular function categories and KEGG pathways. Most of the enriched GO terms and KEGG pathways were related to energy metabolism. Specifically, enriched GO terms included "mitochondrial respiratory chain complex I assembly," "NADH dehydrogenase (ubiquinone) activity," "ATP binding," "mitochondrial translation," "tricarboxylic acid cycle," and "GTP binding" while enriched KEGG pathways included "thermogenesis," "carbon metabolism," and "citrate cycle TCA cycle" (Fig. 5d, e). Mitochondria function as the primary energy producers of the cell and serves as the hub for a variety of immune pathways as the center of biosynthesis, oxidative stress response, and cellular signaling [21]. NADH dehydrogenase is a core subunit of the mitochondrial membrane respiratory chain and is believed to contribute to the minimal assembly required for catalysis [22]. The protein which binds ATP or GTP, carries three phosphate groups esterified to a sugar moiety and provides energy and phosphate sources for the cell $[23,24]$. The tricarboxylic acid cycle is a series of metabolic reactions in aerobic cellular respiration, and is the most important metabolic pathway for the energy supply to the body [25].. The "thermogenesis" pathway is essential for warm-blooded animals, because it ensures normal cellular and physiological functions under challenging environmental conditions [26].

\section{Discussion}

Previous studies described transcription profiling of the mammary gland in livestock (including cattle [27], sheep [28], and goat [29]) and DNA methylation profiling of the mammary gland in cattle [30]. These studies showed temporal and spatial specificity in the methylome and 


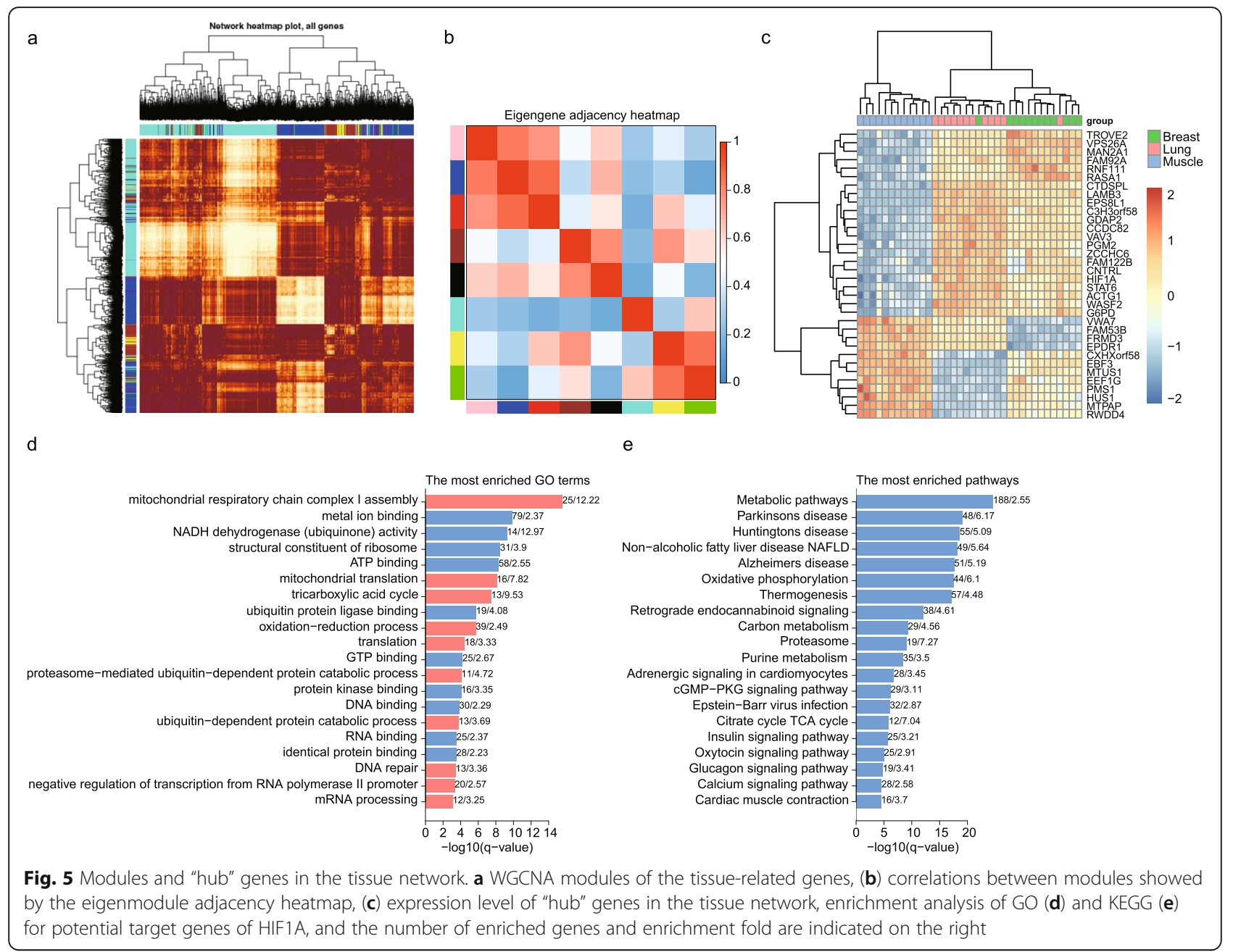

transcriptome profiles of the mammary gland in different species, but they only reported the differential gene expression profile of the mammary gland; and the regulatory network is still unknown. In this study, we for the first time generated the methylomes and transcriptomes of lung, breast, and biceps brachii muscle tissues from yak at four different stages of development $(6,30,54$, and 90 months; young, pre-mature, mature, and postmature, respectively). We found that breast tissue at 90 months showed considerably differential methylation levels compared with other month groups, but lung and biceps brachii muscle tissues did not. Enrichment analysis for upregulated genes with hypomethylated DMRs from breast tissues of 90-month-old yaks showed that DNA methylation might regulate the activation of the "protein processing in endoplasmic reticulum (ER)" pathway. Because only the 90-month-old yaks were in the lactation period, it appears that DNA methylation regulates milk production by influencing protein processing in the endoplasmic reticulum.

In this study, hub genes were identified by WGCNA. The data show that the hub genes with the highest MM and GS in modules of interest should be candidates for further research. This study identified turquoise module genes associated with milk yield, and 20 genes were considered hub genes, showing the highest mRNA expression level in breast tissue at 90 months when yaks enter the lactation period. In these hub genes, ZGPAT was annotated as a transcription factor that potentially regulated the transcription of 280 genes in the "turquoise" module network, which was enriched in the KEGG categories of "aminoacyl-tRNA biosynthesis," "autophagy animal," and "protein processing in endoplasmic reticulum". This result suggests that ZGPAT helps with regulating protein synthesis, processing, and secretion in breast tissue. Moreover, the 7 genes potentially regulated 
Table 2 List of "hub" genes in the consensus network for tissue

\begin{tabular}{|c|c|c|c|c|c|c|c|}
\hline Yak ID & Gene symbol & Module color & Tissue & Gene significance & $p$-value & Module membership & $p$-value \\
\hline BmuPB014336 & EEF1G & turquoise & lung & -0.73907039 & $2.64 \mathrm{E}-07$ & 0.826748126 & $5.20 \mathrm{E}-10$ \\
\hline BmuPB017352 & PMS1 & turquoise & lung & -0.71599797 & $9.13 \mathrm{E}-07$ & 0.850552958 & $5.12 \mathrm{E}-11$ \\
\hline BmuPB000878 & MTPAP & turquoise & lung & -0.715583465 & 9.33E-07 & 0.912877613 & 8.73E-15 \\
\hline BmuPB018762 & CXHXorf58 & turquoise & lung & -0.709433358 & $1.27 \mathrm{E}-06$ & 0.82262518 & 7.50E-10 \\
\hline BmuPB014450 & RWDD4 & turquoise & lung & -0.708526806 & $1.33 \mathrm{E}-06$ & 0.923713618 & $9.94 \mathrm{E}-16$ \\
\hline BmuPB011005 & HUS1 & turquoise & lung & -0.707137304 & $1.43 \mathrm{E}-06$ & 0.875052199 & 2.97E-12 \\
\hline BmuPB005540 & MTUS1 & turquoise & lung & -0.704482832 & 1.62E-06 & 0.871626441 & $4.58 \mathrm{E}-12$ \\
\hline BmuPB011453 & EBF3 & turquoise & lung & -0.703373458 & $1.71 \mathrm{E}-06$ & 0.870717052 & $5.13 \mathrm{E}-12$ \\
\hline BmuPB010608 & LAMB3 & turquoise & lung & 0.70783756 & $1.38 \mathrm{E}-06$ & -0.85067459 & 5.05E-11 \\
\hline BmuPB004299 & C3H3orf58 & turquoise & lung & 0.708872549 & $1.31 \mathrm{E}-06$ & -0.88522675 & 7.61E-13 \\
\hline BmuPB020871 & G6PD & turquoise & lung & 0.708930411 & 1.30E-06 & -0.90727231 & 2.41E-14 \\
\hline BmuPB007438 & ZCCHC6 & turquoise & lung & 0.709740967 & $1.25 \mathrm{E}-06$ & -0.84738754 & 7.13E-11 \\
\hline BmuPB007592 & CTDSPL & turquoise & lung & 0.711634321 & $1.14 \mathrm{E}-06$ & -0.8813581 & 1.30E-12 \\
\hline BmuPB004894 & HIF1A & turquoise & lung & 0.713237417 & $1.05 \mathrm{E}-06$ & -0.87302984 & $3.84 \mathrm{E}-12$ \\
\hline BmuPB020508 & FAM122B & turquoise & lung & 0.720139618 & 7.37E-07 & -0.82428698 & $6.48 \mathrm{E}-10$ \\
\hline BmuPB018102 & CCDC82 & turquoise & lung & 0.720592854 & 7.20E-07 & -0.90665968 & $2.68 \mathrm{E}-14$ \\
\hline BmuPB014539 & CNTRL & turquoise & lung & 0.722130497 & $6.64 \mathrm{E}-07$ & -0.87149974 & 4.65E-12 \\
\hline BmuPB003882 & VAV3 & turquoise & lung & 0.725601285 & 5.53E-07 & -0.89496378 & 1.82E-13 \\
\hline BmuPB020153 & EPS8L1 & turquoise & lung & 0.727495147 & 4.99E-07 & -0.82286844 & 7.34E-10 \\
\hline BmuPB010308 & PGM2 & turquoise & lung & 0.746811608 & 1.69E-07 & -0.83795918 & 1.83E-10 \\
\hline BmuPB004008 & GDAP2 & turquoise & lung & 0.754806874 & 1.05E-07 & -0.88661303 & $6.26 \mathrm{E}-13$ \\
\hline BmuPB012568 & WASF2 & turquoise & lung & 0.757428944 & 8.92E-08 & -0.83874427 & $1.69 \mathrm{E}-10$ \\
\hline BmuPB011966 & ACTG1 & turquoise & lung & 0.772114072 & 3.49E-08 & -0.84373776 & 1.03E-10 \\
\hline BmuPB014173 & STAT6 & turquoise & lung & 0.80896084 & 2.37E-09 & -0.84981355 & $5.53 \mathrm{E}-11$ \\
\hline BmuPB015106 & MAN2A1 & turquoise & breast & 0.705124693 & 1.57E-06 & -0.84782348 & $6.81 \mathrm{E}-11$ \\
\hline BmuPB008614 & VPS26A & turquoise & breast & 0.731553581 & $4.01 \mathrm{E}-07$ & -0.88669366 & $6.18 \mathrm{E}-13$ \\
\hline BmuPB018496 & FAM92A & turquoise & breast & 0.718223061 & $8.14 \mathrm{E}-07$ & -0.85598454 & 2.85E-11 \\
\hline BmuPB005078 & RASA1 & turquoise & breast & 0.705902582 & $1.51 \mathrm{E}-06$ & -0.85869193 & $2.11 \mathrm{E}-11$ \\
\hline BmuPB011476 & FAM53B & turquoise & breast & -0.728187435 & 4.81E-07 & 0.848680696 & $6.23 \mathrm{E}-11$ \\
\hline BmuPB008348 & EPDR1 & turquoise & breast & -0.710771064 & 1.19E-06 & 0.832481139 & 3.08E-10 \\
\hline BmuPB003453 & TROVE2 & turquoise & breast & 0.72654448 & $5.26 \mathrm{E}-07$ & -0.80669368 & 2.84E-09 \\
\hline BmuPB021200 & WWA7 & turquoise & breast & -0.722814498 & $6.41 \mathrm{E}-07$ & 0.814033145 & 1.56E-09 \\
\hline BmuPB010528 & RNF111 & turquoise & breast & 0.737234077 & $2.92 \mathrm{E}-07$ & -0.80146106 & 4.28E-09 \\
\hline BmuPB015811 & FRMD3 & turquoise & breast & -0.739223421 & 2.61E-07 & 0.809147869 & 2.33E-09 \\
\hline
\end{tabular}

by ZGPAT in "protein processing in endoplasmic reticulum" were totally different from the aforementioned 9 genes regulated by hypomethylation, illustrating that DNA methylation and transcription factor possibly co-regulate milk production. In addition, the tissue network analysis confirms the importance of HIF1A in regulating energy metabolism, which is necessary for adaptations to low temperature and hypoxia in high altitudes.

\section{Conclusions}

The results of this comprehensive study provide a solid basis for understanding the roles of DNA methylation and transcriptional network underlying milk protein synthesis and high-altitude adaptation in yaks. This information advances our understanding of the regulatory network in the mammary gland at different developmental stages and may help inform breeding programs aimed at improving milk production. 


\section{Methods}

\section{Animals and samples}

In total, twelve female yaks (belonging to an indigenous yak breed that lives at altitudes of 3800-4000 $\mathrm{m}$ above sea level in Riwoqe, Tibet, China) were collected between June and December of 2016 from private farms (Keqiong farm, Riwoqe, Tibet, China). They were grouped with 3 replicates into age categories of 6, 30, 54, and 90 months. At the time of slaughter, their mean live weights were $44.93 \mathrm{~kg}$ (6 months old), $153.06 \mathrm{~kg}$ (30 months old), $188.3 \mathrm{~kg}$ (54 months old), and $243.56 \mathrm{~kg}$ (90 months old). Only the 90 month-old yaks were lactating, producing $\sim 3.16 \mathrm{~kg} /$ day milk yield $(\sim 120$ days after giving birth for the third time). The 54-month-old yaks were in a dry period ( $~ 540$ days after giving birth for the first time) [31]. The blood relation between the last three generations, which were housed simultaneously and fed the same diets, was unknown. The yaks were not fed the night before they were slaughtered and were humanely sacrificed with the following procedures: (1) showered with clean water close to body temperature $\left(35-38^{\circ} \mathrm{C}\right)$, (2) electrically stunned $(120 \mathrm{~V} \mathrm{dc}, 12 \mathrm{~s})$ prior to exsanguination, (3) sacrificed while in the coma by bloodletting from carotid artery and jugular vein, (4) and dissected rapidly to obtain breast, lung, and biceps brachii muscle tissue samples, which were immediately frozen in liquid nitrogen, and stored at $-80^{\circ} \mathrm{C}$ until RNA and DNA extraction.

\section{Whole genome bisulfite sequencing}

The QIAamp DNA Mini Kit (Qiagen, Hilden, Germany) was used to isolate the high-quality DNA from each sample. According to the manufacturer's instructions, $1 \mu \mathrm{g}$ of genomic DNA was fragmented by sonication to a mean size of approximately $250 \mathrm{bp}$ and subsequently used for whole genome bisulfite sequencing (WGBS) library construction using an Acegen Bisulfite-Seq Library Prep Kit (Acegen, Shenzhen, GD, China). Briefly, fragmented DNA was end-repaired, 5'-phosphorylated, 3'$\mathrm{dA}$-tailed, and then ligated to methylated adapters. The methylated adapter-ligated DNAs were purified using $1 \times$ Agencourt AMPure XP magnetic beads (Beckman Coulter, Brea, CA, USA) and subjected to bisulfite conversion with a ZYMO EZ DNA Methylation-Gold Kit (Zymo research, Irvine, CA, USA). The converted DNAs were then amplified using $25 \mu \mathrm{l} \mathrm{HiFi} \mathrm{HotStart} \mathrm{U+} \mathrm{RM}$ and 8-bp index primers with a final concentration of $1 \mu \mathrm{M}$ each. The constructed WGBS libraries were then analyzed with an Agilent 2100 Bioanalyzer (Agilent Technologies, SantaClara, CA, USA), quantified with a Qubit fluorometer with Quant-iT dsDNA HS Assay Kit (Invitrogen, Carlsbad, CA,USA), and finally sequenced on an Illumina Hiseq $\mathrm{X}$ ten sequencer (PE150 mode) (Illumina, San Diego, CA, USA).

\section{Methylation calculation and identification of DMRs}

Low quality reads that contained more than 5 'N's or had a low-quality value for over $50 \%$ of the sequence (Phred score $<5)$ were filtered. The sequencing reads of the samples were aligned to the yak reference genome [32] using BSMAP (Version 2.74) [33]. The methylated CpG (mCG) sites were identified following a previously described algorithm [34]. The methylation levels for each sample were calculated using in-house Perl scripts. Differentially methylated regions (DMRs) were identified using metilene (Version 0.2-6) within a $500 \mathrm{bp}$ sliding window at $250 \mathrm{bp}$ steps with at least $10 \mathrm{CpGs}$ covered by over $10 \times$ sequence reads, applying the thresholds of differential methylation $\beta>=15 \%$, FDR for two-dimensional KolmogorovSmirnov-Test $p$-value $<0.05$ [35].. The enrichment analyses were conducted using WebGestalt (WEB-based Gene SeT AnaLysis Toolkit) [36].

Total RNA extraction, library preparation, and sequencing The TRIzol reagent (Invitrogen, Carlsbad, CA, USA) was used to isolate the total RNA of each sample. The purity, concentration, and integrity of RNA were checked using the NanoPhotometer spectrophotometer (IMPLEN, Westlake Village, CA, USA), the Qubit RNA Assay Kit in Qubit 2.0 Fluorometer (Life Technologies, Carlsbad, CA, USA), and the RNA Nano 6000 Assay Kit of the Bioanalyzer 2100 System (Agilent Technologies, SantaClara, CA, USA), respectively. For each sample, $3 \mu \mathrm{g}$ high-quality RNA was used as input material for RNAseq library preparation. First, ribosomal RNA was removed using the Epicentre Ribo-Zero rRNA Removal Kit (Epicentre, Madison, WI, USA). Next, the rRNAdepleted RNA was used to create sequencing libraries using the NEBNext Ultra Directional RNA Library Prep Kit for Illumina (NEB, Ipswich, MA, USA). Finally, the library products were purified using $1 \times$ Agencourt AMPure XP magnetic beads (Beckman Coulter, Brea, CA, USA) and the Agilent Bioanalyzer 2100 System (Agilent Technologies, SantaClara, CA, USA) was employed to assess the library quality. Clustering of the index-coded samples was completed on a cBot Cluster Generation System using the TruSeq PE Cluster Kit v3cBot-HS (Illumina, San Diego, CA, USA), and then the libraries were sequenced on the Illumina HiSeq $\mathrm{X}$ Ten Platform to generate $150 \mathrm{bp}$ paired-end reads.

\section{Quality analysis, transcriptome assembly, and abundance} estimation

Clean reads were obtained by removing reads containing the adapter or poly- $\mathrm{N}$ and by removing low quality reads (over $10 \%$ of the sequence with a quality value <30) from the raw data using in-house Perl scripts. All downstream analyses were based on the good-quality clean reads. Paired-end clean reads were mapped to the yak 
reference genome [32] with STAR (available at https:// github.com/alexdobin/STAR/releases). The mapped reads of each sample were assembled using StringTie [37]. Next, all sample transcriptomes were merged to reconstruct a comprehensive transcriptome using Perl scripts. After the final transcriptome was generated, StringTie and edgeR were used to estimate the expression levels of all transcripts [38]. StringTie was used to assess the expression level of mRNAs by calculating fragments per kilobase of transcript per million fragments mapped (FPKM). Differentially expressed mRNAs were identified using the DESeq2 package, with the criteria of fold-change $\log 2>1$ or $\log 2<-1$ and with the statistical significance set to FDR $<0.05$.

\section{Weighted gene correlation network analysis}

WGCNA networks was generated for both age-related and tissue-related genes, following the overall approach described by Langfelder et al. [14]. Briefly, gene coexpression network was constructed based on a signed Spearman correlation; after network construction, modules are defined as clusters of densely interconnected genes by the topological overlap metric (TOM) and the dynamic tree cut algorithm [15] with a height of 0.25 and a deep split level of 2, a reassign threshold of 0.2 , and a minimum module size of 30 (100 for the consensus network); module relationships were studied by eigenmodules-the first principal component of the module and a signature of gene expression, and each module that was correlated with the dose-response curve with a $p$-value $<0.01$ was considered statistically significant.

\section{Supplementary information}

Supplementary information accompanies this paper at https://doi.org/10. 1186/s12864-020-07151-3.

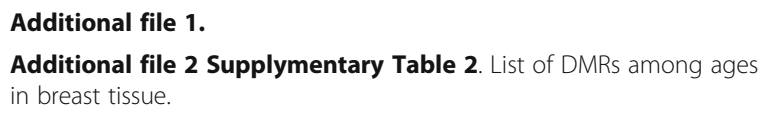

Additional file 6 Supplymentary Table $\mathbf{6}$. WGCNA analysis of tissuerelated genes.

\footnotetext{
Abbreviations

QTP: Qinghai-Tibetan Plateau; DMRs: Differentially methylated regions; WGBS: Whole genome bisulfite sequencing; FPKM: Fragments per kilobase of transcript per million fragments; WGCNA: Weighted gene correlation network analysis; TOM: Topological overlap metric; GO: Gene ontology; KEGG: Kyoto encyclopedia of genes and genomes; A-DMRs: Age-related DMRs; ER: Endoplasmic reticulum; GS: Gene significance; MM: Module membership measures
}

\section{Acknowledgements}

The authors thank the animal husbandry station of Chang Du and the agricultural bureau of Riwoge for all their support.

\section{Authors' contributions}

$J X, Q J$ and JZ planned and coordinated the study and wrote the manuscript. $Z C, C Z, Y C, X C$ and $H J$ collected the samples and performed the library construction, sequencing and the quality control analysis. QZ, YZ and $\mathrm{HC}$ performed downstream analysis of the data and assisted in the generation of additional files for the manuscript. All authors read and approved the final manuscript.

\section{Funding}

This work was supported by a program of Provincial Department of Finance of the Tibet Autonomous Region (No: XZNKY-2019-C-052), the Key Research and Development Projects in Tibet : Preservation of Characteristic Biological Germplasm Resources and Utilization of Gene Technology in Tibet (Grant No. XZ202001ZY0016N ), the Second Tibetan Plateau Scientific Expedition and Research Program (STEP) (Grant No. 2019QZKK0501), Program National Beef Cattle and Yak Industrial Technology System (No: CARS-37), Basic Research Programs of Sichuan Province (No: 2019YJ0256) and the Open Project Program of State Key Laboratory of Hulless Barley and Yak Germplasm Resources and Genetic Improvement (NO:XZNKY-2019-C-007 K10). The funding bodies had no role in the study design; collection, analysis, and interpretation of data; or in writing the manuscript.

\section{Availability of data and materials}

The DNA methylation data and RNA transcriptome data in this study are available in SRA database under the accession numbers PRJNA530286 and PRJNA512958, respectively. The yak reference genome is deposited in Dryad database (https://datadryad.org/stash/share/-99YgHwMBNaAWlo1Tw_ BRcIXHnpYq2ST4iZZpEE7bc).

\section{Ethics approval and consent to participate}

The whole study and protocols for collection of the semen samples of yaks were reviewed and approved by the Ethics Committee at Institute of Animal Science and Veterinary, Tibet Academy of Agricultural and Animal Husbandry Sciences (Permit Number: 2015-216). Written informed consent was obtained from the farm owners.

\section{Consent for publication}

Not applicable.

\section{Competing interests}

The authors declare that they have no competing interests.

\section{Author details}

${ }^{1}$ State Key Laboratory of Hulless Barley and Yak Germplasm Resources and Genetic Improvement, Lhasa, Tibet, China. ${ }^{2}$ Institute of Animal Science and Veterinary, Tibet Academy of Agricultural and Animal Husbandry Sciences, Lhasa, Tibet, China. ${ }^{3}$ Key Laboratory of Qinghai-Tibetan Plateau Animal Genetic Resource Reservation and Utilization, Sichuan Province and Ministry of Education, Southwest Minzu University, Chengdu, Sichuan, China.

Received: 21 January 2020 Accepted: 14 October 2020

Published online: 20 October 2020

\section{References}

1. Wiener G, Han J-L, Long R-J. The yak. 2nd ed. Bangkok, Thailand: Regional Office for Asia and the Pacific, Food and Agriculture Organization of the United Nations; 2003.

2. Qiu Q, Zhang G, Ma T, Qian W, Wang J, Ye Z, Cao C, Hu Q, Kim J, Larkin DM. The yak genome and adaptation to life at high altitude. Nat Genet. 2012; 44(8):946-9.

3. Nichols K, Doelman J, Kim JJM, Carson M, Metcalf JA, Cant JP. Exogenous essential amino acids stimulate an adaptive unfolded protein response in the mammary glands of lactating cows. J Dairy Sci. 2017;100(7):5909-21.

4. Kanwar JR, Kanwar RK, Sun X, Punj V, Matta H, Morley SM, Parratt A, Puri M, Sehgal R. Molecular and biotechnological advances in milk proteins in relation to human health. Curr Protein Pept Sci. 2009;10(4):308-38. 
5. Osorio JS, Lohakare J, Bionaz M. Biosynthesis of milk fat, protein, and lactose: roles of transcriptional and posttranscriptional regulation. Physiol Genomics. 2016;48(4):231-56.

6. Gene Ontology Consortium. Expansion of the gene ontology knowledgebase and resources. Nucleic Acids Res. 2017;45(D1):D331-8.

7. Finn RD, Attwood TK, Babbitt PC, Bateman A, Bork P, Bridge AJ, Chang HY, Dosztányi Z, El-Gebali S, Fraser M, Gough J, Haft D, Holliday GL, Huang H, Huang X, Letunic I, Lopez R, Lu S, Marchler-Bauer A, Mi H, Mistry J, Natale DA, Necci M, Nuka G, Orengo CA, Park Y, Pesseat S, Piovesan D, Potter SC, Rawlings ND, Redaschi N, Richardson L, Rivoire C, Sangrador-Vegas A, Sigrist C, Sillitoe I, Smithers B, Squizzato S, Sutton G, Thanki N, Thomas PD, Tosatto SC, Wu CH, Xenarios I, Yeh LS, Young SY, Mitchell AL. InterPro in 2017beyond protein family and domain annotations. Nucleic Acids Res. 2017; 45(D1):D190-9.

8. Kanehisa M, Furumichi M, Tanabe M, Sato Y, Morishima K. KEGG: new perspectives on genomes, pathways, diseases and drugs. Nucleic Acids Res. 2017;45(D1):D353-61.

9. Boutet $E$, Lieberherr $D$, Tognolli $M$, Schneider $M$, Bansal P, Bridge AJ, Poux S, Bougueleret L, Xenarios I. UniProtKB/Swiss-Prot, the manually annotated section of the UniProt KnowledgeBase: how to use the entry view. Methods Mol Biol. 2016;1374:23-54.

10. Junker V, Contrino S, Fleischmann W, Hermjakob H, Lang F, Magrane M, Martin MJ, Mitaritonna N, O'Donovan C, Apweiler R. The role SWISS-PROT and TrEMBL play in the genome research environment. J Biotechnol. 2000; 78(3):221-34.

11. Burgess, D.J: Gene expression: principles of gene regulation across tissues. Nat Rev Genet, 2017, 18(12):701-701.

12. Preston $\mathrm{GM}$, Brodsky JL. The evolving role of ubiquitin modification in endoplasmic reticulum-associated degradation. Biochem J. 2017:474(4):44569.

13. McCaffrey K, Braakman I. Protein quality control at the endoplasmic reticulum. Essays Biochem. 2016;60(2):227-35.

14. Langfelder P, Horvath S. WGCNA: an R package for weighted correlation network analysis. BMC Bioinformatics. 2008;9:559.

15. Liu X, Hu AX, Zhao JL, Chen FL. Identification of key gene modules in human osteosarcoma by co-expression analysis weighted gene coexpression network analysis (WGCNA). J Cell Biochem. 2017;118(11):3953-9.

16. Hu H, Miao YR, Jia LH, Yu QY, Zhang Q, Guo AY. AnimalTFDB 3.0: a comprehensive resource for annotation and prediction of animal transcription factors. Nucleic Acids Res. 2019;7(D1):D33-8.

17. Sun LD, Xiao FL, Li Y, Zhou WM, Tang HY, Tang XF, Zhang H, Schaarschmidt H, Zuo XB, Foelster-Holst R, He SM, Shi M, Liu Q, Lv YM, Chen XL, Zhu K, Guo YF, Hu DY, Li M, Li M, Zhang YH, Zhang X, Tang JP, Guo BR, Wang H, Liu Y, Zou XY, Zhou FS, Liu XY, Chen G, Ma L, Zhang SM, Jiang AP, Zheng $X D$, Gao XH, Li P, Tu CX, Yin XY, Han XP, Ren YQ, Song SP, Lu ZY, Zhang XL, Cui Y, Chang J, Gao M, Luo XY, Wang PG, Dai X, Su W, Li H, Shen CP, Liu SX, Feng XB, Yang CJ, Lin GS, Wang ZX, Huang JQ, Fan X, Wang $Y$, Bao YX, Yang S, Liu JJ, Franke A, Weidinger S, Yao ZR, Zhang XJ. Genome-wide association study identifies two new susceptibility loci for atopic dermatitis in the Chinese Han population. Nat Genet. 2011;43(7):690-4.

18. Liao D. Emerging roles of the EBF family of transcription factors in tumor suppression. Mol Cancer Res. 2009;7(12):1893-901.

19. Jargosch M, Kröger S, Gralinska E, Klotz U, Fang Z, Chen W, Leser U, Selbig J, Groth D, Baumgrass R. Data integration for identification of important transcription factors of STAT6-mediated cell fate decisions. Genet Mol Res. 2016;15(2). https://doi.org/10.4238/gmr.15028493.

20. Moniz S, Biddlestone J, Rocha S. Grow2: the HIF system, energy homeostasis and the cell cycle. Histol Histopathol. 2014;29(5):589-600.

21. van der Bliek AM, Sedensky MM, Morgan PG. Cell biology of the mitochondrion. Genetics. 2017;207(3):843-71.

22. Ganesan V, Sivanesan D, Yoon S. Correlation between the structure and catalytic activity of [Cp*Rh (substituted Bipyridine)] complexes for NADH regeneration. Inorg Chem. 2017;56(3):1366-74.

23. Li T, Liu J, Smith WW. Synphilin-1 binds ATP and regulates intracellular energy status. PLoS One. 2014;9(12):e115233.

24. Goody RS. The significance of the free energy of hydrolysis of GTP for signal-transducing and regulatory GTPases. Biophys Chem. 2003;100(1-3): 535-44

25. Nunes-Nesi A, Araújo WL, Obata T, Fernie AR. Regulation of the mitochondrial tricarboxylic acid cycle. Curr Opin Plant Biol. 2013;16(3):335-43.
26. Bal NC, Singh S, Reis FCG, Maurya SK, Pani S, Rowland LA, Periasamy M. Both brown adipose tissue and skeletal muscle thermogenesis processes are activated during mild to severe cold adaptation in mice. J Biol Chem. 2017;292(40):16616-25.

27. Cui X, Hou Y, Yang S, Xie Y, Zhang S, Zhang Y, Zhang Q, Lu X, Liu GE, Sun D. Transcriptional profiling of mammary gland in Holstein cows with extremely different milk protein and fat percentage using RNA sequencing. BMC Genomics. 2014;15:226.

28. Suárez-Vega A, Gutiérrez-Gil B, Klopp C, Tosser-Klopp G, Arranz JJ. Comprehensive RNA-Seq profiling to evaluate lactating sheep mammary gland transcriptome. Sci Data. 2016;3:160051.

29. Mobuchon L, Marthey S, Boussaha M, Le Guillou S, Leroux C, Le Provost F. Annotation of the goat genome using next generation sequencing of microRNA expressed by the lactating mammary gland: comparison of three approaches. BMC Genomics. 2015;16(1):285.

30. Yang Z, Connor EE, Bickhart DM, Li C, Baldwin RL, Schroeder SG, Rosen BD, Yang L, Van Tassell CP, Liu GE. Comparative whole genome DNA methylation profiling of cattle sperm and somatic tissues reveals striking hypomethylated patterns in sperm. Gigascience. 2018;7(5):giy039.

31. Li Z, Jiang M. Metabolomic profiles in yak mammary gland tissue during the lactation cycle. PLoS One. 2019;14(7):e0219220.

32. Ji QM, Xin JW, Chai ZX, Zhang CF, Dawa Y, Luo S, Zhang Q, Pingcuo Z, Peng MS, Zhu Y, Cao HW, Wang H, Jian-Lin H, Zhong JC. Achromosomescale reference genome and genome-wide genetic variations elucidate adaptation in yak. Mol Ecol Resour. 2020. https://doi.org/10.1111/1755-0998. 13236.

33. Xi Y, Li W. BSMAP: whole genome bisulfite sequence MAPping program. BMC Bioinformatics. 2009;10(1):232

34. Lister R, Pelizzola M, Dowen RH, Hawkins RD, Hon GC, Tontifilippini J, Nery JR, Lee LK, Ye Z, Ngo Q. Human DNA methylomes at base resolution show widespread epigenomic differences. Nature. 2009;462(7271):315-22.

35. Juhling F, Kretzmer H, Bernhart SH, Otto C, Stadler PF, Hoffmann S. Metilene: fast and sensitive calling of differentially methylated regions from bisulfite sequencing data. Genome Res. 2016;26(2):256-62.

36. Wang J, Duncan D, Shi Z, Zhang B. WEB-based GEne SeT AnaLysis toolkit (WebGestalt): update. Nucleic Acids Res. 2013;41:W77-83.

37. Pertea M, Pertea G, Antonescu C, Chang TC, Mendell JT, Salzberg SL. StringTie enables improved reconstruction of a transcriptome from RNA-seq reads. Nat Biotechnol. 2015;33(3):290-5.

38. Robinson MD, Mccarthy DJ, Smyth GK. edgeR: a bioconductor package for differential expression analysis of digital gene expression data. Bioinformatics. 2010;26(1):139-40.

\section{Publisher's Note}

Springer Nature remains neutral with regard to jurisdictional claims in published maps and institutional affiliations.
Ready to submit your research? Choose BMC and benefit from:

- fast, convenient online submission

- thorough peer review by experienced researchers in your field

- rapid publication on acceptance

- support for research data, including large and complex data types

- gold Open Access which fosters wider collaboration and increased citations

- maximum visibility for your research: over $100 \mathrm{M}$ website views per year

At $\mathrm{BMC}$, research is always in progress.

Learn more biomedcentral.com/submission 